\title{
FUNDAMENTOS TEÓRICO - METODOLÓGICOS PARA LA FORMACIÓN EN EMPRENDIMIENTO Y EL DESARROLLO DE LA CAPACIDAD DE INNOVACIÓN EN PROFESIONALES. CASO DE ESTUDIO: FACULTAD DE ESPECIALIDADES EMPRESARIALES, UCSG
}

\author{
THEORETICAL-METHODOLOGICAL FOUNDATIONS FOR TRAINING IN \\ ENTREPRENEURSHIP AND DEVELOPMENT OF INNOVATION CAPACITY IN \\ PROFESSIONALS. CASE STUDY: SPECIALTIES FACULTY OF BUSINESS, UCSG
}

LUIS FERNANDO HIDALGO PROAÑO

Universidad Católica de Santiago de Guayaquil

luis.hidalgo01@cu.ucsg.edu.ec

RESUMEN

Este artículo presenta la sustentación teórica y metodológica para un proyecto de formación en emprendimiento y fortalecimiento de capacidad de innovación de profesionales que toma como caso de estudio la carrera de Emprendedores de la Universidad Católica de Santiago de Guayaquil. El tema que aborda reviste una particular importancia, dado que el fortalecimiento de propuestas emprendedoras constituyen hoy una necesidad para el cambio de la matriz productiva y el desarrollo del país, de ahí la pertinencia de la formación en esa área que dotaría a los graduados de capacidades como pensamiento estratégico, conciencia de la necesidad de innovación, preparación para enfrentar el cambio y la incertidumbre, competencias comunicativas y capacidad para la identificación de nuevas necesidades, por sólo señalar algunas de las ventajas que se derivarían de esa formación. La importancia de la investigación se relaciona con el aporte que supone una fundamentación tanto teórica como metodológica acerca de la pertinencia de un proyecto educativo para la formación de profesionales emprendedores, lo cual podría influir en la incorporación de este tipo de formación en diversas carreras universitarias y con ello contribuir a una mejor inserción de los graduados en la sociedad, así como a un mayor aporte por su parte en cuanto al desarrollo de sus comunidades y por ende, del país.
PALABRAS CLAVE: emprendedor, innovación, formación de emprendedores, transformación, desarrollo.
This paper presents the theoretical and methodological support for a project training in entrepreneurship and strengthening of innovation capacity of professionals, it takes as a case study the career of Entrepreneurs of the Catholic University of Santiago de Guayaquil. The topic discussed is particularly important, since the strengthening of entrepreneurial proposals constitute a need for the change of the productive matrix and the country's development, thus the relevance of training in that area that would provide graduates capabilities such as strategic thinking, awareness of the need for innovation, preparation to face change and uncertainty, communication skills and ability to identify new needs, only point out some of the advantages that would result from that training.The importance of this research relates to the contribution which represents a theoretical basis and methodological about the relevance of an educational project for the training of entrepreneurs professionals, which could influence the incorporation of this kind of training in various university courses and thereby contributing to a better integration of graduates in society, as well as a greater contribution in the development of their communities and therefore, their country.

KEYWORDS: entrepreneurship, innovation, entrepreneurship training, transformation, development. 


\section{INTRODUCCIÓN}

No se ha realizado hasta nuestros días un estudio similar. La presente ponencia presenta la sustentación teórica y metodológica para un proyecto de formación en emprendimiento y fortalecimiento en capacidad de innovación de futuros graduados, que toma como caso de estudio la carrera de Emprendedores de la Universidad Católica de Santiago de Guayaquil.

El tema que aborda reviste una particular importancia, dado que el fortalecimiento de propuestas emprendedoras constituye hoy una necesidad para el cambio de la matriz productiva y el desarrollo del país; de ahí la pertinencia de la formación en esa área que dotaría a los graduados de capacidades como pensamiento estratégico, conciencia de la necesidad de innovación, preparación para enfrentar el cambio y la incertidumbre; competencias comunicativas y capacidad para la identificación de nuevas necesidades, por sólo señalar algunas de las ventajas que se derivarían de esa formación. El emprendimiento es una necesidad para el cambio de la matriz productiva y el desarrollo del país.

El limitado crecimiento de la pequeña y mediana empresa, de corte nacional, es uno de los aspectos que inciden decisivamente en el insuficiente desarrollo de los países de América Latina, así lo reconocen destacados especialistas, (CEPAL 2012; Buitelaar, 2010; Zevayos, 2004, entre otros). Frente a las tendencias globalizadoras de los últimos años, en condiciones de desigual competencia, las empresas nacionales pequeñas y medianas perecen, y con ellas se extinguen también productos y servicios que se relacionaban directamente con usos culturales y modos de vidas propios.

No por casualidad desde finales del Siglo XX y principios de este, se han realizado reiterados llamados por parte de organismos internacionales, como la Comisión Económica para América Latina (CEPAL) a fin de que se tomen medidas tendientes a proteger a la pequeña y mediana empresa nacional, (PYMES en adelante), a la vez que se subraya en múltiples foros su importancia económica y social. (CEPAL, 2012).

Se convoca en la actualidad a las empresas en América Latina a la innovación, a la generación de empleos y al aumento de productividad, y más recientemente a la integración, aprovechando los cambios del panorama latinoamericano que la favorecen.

\section{HACIA EL EMPRENDIMIENTO Y LA INNOVACIÓN EN EL} ECUADOR. OPORTUNIDADES Y AMENAZAS

Esta situación se ajusta al escenario ecuatoriano actual, en el cual se ofrecen posibilidades para el despegue de este tipo de entidad, de tal forma que se pudiera afirmar que hoy en Ecuador, en sentido general, y en Guayaquil en particular, las PYMES constituyen un espacio deseable, oportuno, factible para satisfacer necesidades relacionadas con el cambio de la matriz productiva del país, objetivo estratégico de primer orden en el Plan Nacional de Desarrollo para el Buen Vivir en su etapa del 2013 al 2017.

Existen determinadas limitaciones reales que frenan el crecimiento y desarrollo de las empresas pequeñas y medianas, entre las cuales se puede citar la falta de preparación para su surgimiento, su creación intuitiva, sin estudio previo, sin aplicación de un pensamiento estratégico y, por tanto, privadas de posibilidades de innovación y adaptación al cambio, que podrían atenuarse mediante la formación de profesionales emprendedores, dotados de los conocimientos y competencias que reducirían estas

LIMITACIONES DE MANERA SUSTANCIAL

Entre ellas podría citarse la insuficiente respuesta de la academia en cuanto a la formación en administración de negocios en el Ecuador, la cual carece en muchos casos de ese enfoque. Se sigue formando a los futuros administradores o creadores de empresas en el país en carreras de corte administrativo tradicionales, que no preparan a los futuros graduados para la identificación de necesidades de productos y servicios en la sociedad y sobre todo en la posibilidad de responder a estas necesidades mediante la innovación o la creación de nuevas empresas.

Según las investigadoras ecuatorianas Arteaga y Lasio (2009:13): "la educación en emprendimiento y creación de negocios incide favorablemente en la intención de emprender, especialmente de aquellos emprendedores que se capacitaron durante sus estudios primarios y secundarios; y además, que el estar expuesto a algún tipo de educación en Emprendimiento, influye en la percepción de poseer conocimientos y habilidades necesarias para crear un negocio". Pero no se da este tipo de formación con la amplitud y profundidad necesaria en las universidades del Ecuador en la actualidad.

De ahí la importancia de formar Emprendedores que serán capaces de identificar oportunidades, analizar recursos y evaluar ideas para implementar proyectos que se concreten 
en nuevos negocios; e innovar o reinventar negocios existentes que se encuentren en etapa de estabilización por lo que estará preparado para trabajar en sus propias empresas jurídicas y en áreas gerenciales de cualquier ámbito.

LA FORMACIÓN EN EMPRENDIMIENTO: FUNDAMENTOS, EVOLUCIÓN Y PROYECCIONES EN EL ECUADOR

Una de las primeras definiciones sobre el emprendedor lo caracteriza como aquella persona que empieza una nueva actividad, ya sea económica, social o política." (Hidalgo y Castro, 2012: 62). Timmons (1989) relaciona el concepto con la realización de acciones por parte de seres humanos creativos para construir algo de valor a partir del aprovechamiento de oportunidades donde aparentemente no existían posibilidades para hacerlo.

El emprendimiento apunta a maximizar las oportunidades. Atendiendo a esto, autores como Drucker (1998) priorizan el logro de efectividad, mientras otros, como Timmons, centran su atención en la búsqueda de las oportunidades. A finales de los noventa, el tema de la innovación, la producción y los contextos en relación con el emprendimiento es abordado por Audretsch (1996), que destaca una trilogía de conceptos: el ser humano como agente innovador, la innovación tecnológica y el mejoramiento continuo.

Uno de los referentes más conocidos es el modelo de Timmons, que asume como factores fundamentales el emprendedor, la oportunidad y los recursos y el equipo. La integración de todos los elementos de forma equilibrada es lo que posibilita la creación de nuevos negocios, lograr ese equilibrio es función del emprendedor.

Las experiencias de formación en emprendimiento en sentido general se orientan hacia la formación mediante materias aisladas, cursos que se insertan en las carreras, proyectos en los que participan estudiantes vacacionados, y experiencias de centro denominados "incubadoras" de empresas.

La carrera de emprendedores de la UCSG, en cambio, se ha diseñado para la formación en emprendimiento con la amplitud y la profundidad que propicia una carrera universitaria, la cual en sus años de vida, ha ido perfeccionando su gestión y fortaleciendo sus relaciones con los sectores sociales con los que se vincula.

Vinculado con la necesidad de perfeccionar este tipo de formación, se planteó como problema de investigación: ¿Cómo promover la formación de profesionales emprendedores, capaces de diseñar e implementar PYMES que contribuyan a la transformación de la matriz productiva del país?

A partir de lo cual se determinó como objetivo general: Fundamentar teórica y metodológicamente un Proyecto Educativo para la Formación en Emprendimiento en la facultad de Especialidades Empresariales (FEE) de la UCSG. Y entre los bjetivos específicos se destacan:

1. Caracterizar la situación que presenta hoy la formación de emprendedores como carrera de la FEE-USCG así como la inserción de sus graduados en el sector empresarial de la ciudad de Guayaquil y explorar las posibilidades de implantación de este tipo de formación como eje transversal en las restantes carreras de la FEE-USCG.

2. Fundamentar los elementos conceptuales que constituyen el basamento teórico de la formación en emprendimiento como idea rectora de la carrera de estudio y como eje transversal de formación en las restantes carreras de la facultad de Especialidades Empresariales

3. Formular las bases metodológicas del proyecto educativo para la formación en emprendimiento en la facultad de Especialidades Empresariales.

4. Validar, mediante criterio de expertos, la pertinencia, calidad y factibilidad de la fundamentación teórico-metodológica que se propone.

En la indagación se emplearon métodos teóricos (análisis y síntesis, comparación, histórico- lógico), que posibilitaron el análisis del estado del arte, el estudio comparativo de las tendencias teóricas que sustentan la formación de emprendedores, así como la generalización y síntesis de las propuestas más importantes en este tema. A los efectos de la construcción de la fundamentación se aplicaron igualmente otros métodos teóricos como la inducción y la deducción en la interpretación de resultados y la determinación de los modos de desarrollar esa formación a partir de sus bases teóricas y metodológicas.

Se emplearon además métodos empíricos para la búsqueda de información necesaria en las diversas fuentes seleccionadas, a los que se aplicaron encuestas y entrevistas en profundidad. De este modo pudo lograrse una triangulación de datos aportados por diversas técnicas y fuentes, que posibilitó una mirada con amplio espectro. 
Las técnicas descritas se aplicaron a muestras de graduados seleccionados, profesionales, autoridades, y profesores, los cuales constituyeron unidades de análisis; (ver Anexo 1). Por razones de limitación de espacio no resulta posible referirse a los resultados obtenidos como respuesta a cada uno de los objetivos, por tanto se presentarán los elementos fundamentales de la propuesta que constituyó el centro de interés de la tesis doctoral.

ELEMENTOS FUNDAMENTALES DEL PROYECTO PROPUESTO

Para la construcción del proyecto se tomó como punto de partida la categoría de análisis, fundamentación teórico metodológica de la formación en emprendimiento, estudiada en dos dimensiones: dimensión 1, teórica-conceptual; las bases teóricas de la formación en emprendimiento; y dimensión 2, metodológica-instrumental: las bases operacionales de la formación en emprendimiento.

Estas dimensiones están presentes en los dos escenarios del Proyecto Educativo; el primero, como idea rectora de la carrera de Emprendedores; el segundo, como eje transversal de las restantes carreras de dicha facultad.

De esta forma las bases conceptuales de la formación en emprendimiento se constituyen en plataforma teórica para el primer escenario del Proyecto Educativo, la formación en emprendimiento como idea rectora de la carrera de Emprendedores y, por tanto, sus componentes epistemológicos a nivel de definiciones, conceptos, categorías, principios y fuentes de argumentación pasan a integrar el sistema de conocimientos de esta formación. La dimensión metodológica-instrumental, bases operacionales de la formación de emprendedores, se expone a continuación mediante la presentación del proyecto en sus diversas partes, componentes y fases.

El proyecto se define por su carácter emprendedor, integrador y consensuado, flexible y dinámico, vinculante. Otros rasgos que lo identifican son: el carácter sistémico, la articulación entre sus componentes y la orientación a la búsqueda de calidad. Su finalidad es la de perfeccionar la formación integral de los profesionales de la facultad de Especialidades Empresariales, a través del fortalecimiento de su cultura de emprendimiento, y su capacidad de innovación, lo cual se expresa en dos dimensiones: como idea rectora de la carrera de Emprendedores y como eje transversal de las restantes carreras de dicha Facultad.
El proyecto educativo asume, en primer lugar, los principios declarados por la LOES y por la UCSG, a saber: los principios de calidad, pertinencia, integralidad y autodeterminación para la producción del pensamiento y conocimiento. A estos principios el autor de la tesis adiciona: el Principio del Fomento de la Cultura de emprendimiento y el Principio de la Participación, cooperación y trabajo en equipo.

El concepto de idea rectora expresa una relación determinante con el perfil profesional del egresado y con sus modos de actuación profesional, por lo que el contenido que se asume como tal es decisivo en el diseño curricular de la carrera, y todas las disciplinas y asignaturas, como todas las actividades de los componentes investigativo, de práctica y de vinculación social se subordinan a él.

El concepto de eje transversal expresa una relación mucho más flexible, como conocimiento general, como componente cultural y educativo dentro de la formación de un profesional, que se trabaja a través de diferentes asignaturas del currículo, con diferentes grados de implicación, que no está directamente relacionado con el perfil profesional ni con los modos de actuación.

En el proyecto se definen los ámbitos en los que debe implementarse: el de socialización, orientado a compartir resultados y elementos centrales del proyecto entre los actores involucrados en él, como estudiantes, docentes y directivos, por sólo mencionar algunos, organización metodológica, investigación, Vinculación, procesos medulares de la formación universitaria, y capacitación, dirigida a docentes involucrados en este tipo de formación procedentes de otras carreras. Los campos con que se relacionan algunas de las acciones propuestas en el ámbito de la formación son los que están establecidos como parte del plan de estudios por el Reglamento del Régimen Académico de la UCSG. (2013), (ver Anexo 2).

Componente indispensable del proyecto es el sistema de control y la evaluación de los resultados. De hecho, el control sistemático y la evaluación frecuente de cada una de las acciones previstas en el proyecto es lo que permite una valoración objetiva del cumplimiento de los objetivos, corregir las desviaciones o deficiencias, potenciar los éxitos y avances y registrar las buenas prácticas que garantizan la sostenibilidad en el tiempo.

Para la validación de la propuesta se aplicó el método Delphi, con 25 expertos cuyo coeficiente de experticia fue de 0,91 . Estos expertos evalua- 
ron la fundamentación teórico metodológica del proyecto mediante enunciados que se referían a sus dos dimensiones.

En cuanto a la primera: fundamentación teórica conceptual, el $84 \%$ de los expertos seleccionados opinaron que el proyecto tiene una estructura teórica- conceptual muy bien integrada. (Ver Anexo 3). En lo que respecta a la segunda: metodológica- operacional, los resultados fueron aún más favorables. El 65.5\% en promedio de los expertos opinaron que están completamente de acuerdo (CA) en que el proyecto de formación de emprendedores contiene una base metodológica que permite desarrollar las habilidades y destrezas en los formandos. (Ver Anexo 3).

Los valores obtenidos por cada una de las dimensiones son demostrativos de un alto nivel de coincidencia positiva entre los expertos, que en promedio obtuvieron 4,3 puntos sobre 5 en la dimensión teórica y 4,4 en la dimensión metodológica, con las mayores calificaciones en cuanto a la adecuación a las necesidades económicas $(4,7$ sobre 5$)$ y su pertinencia como componente dentro del proyecto educativo (4,6 sobre 5). (Véase Anexo 3).

El total de los resultados promedio de las dos dimensiones a validar de la fundamentación teórica y metodológica del proyecto educativo de formación de emprendedores evidencia que el $61.4 \%$ de los expertos consultados opinaron estar completamente de acuerdo (CA) y el $26 \%$ muy de acuerdo (MA) con ellas, lo que integrando estos resultados positivos muestra un porcentaje de aceptación del $87.4 \%$ en promedio, lo que permite validar la pertinencia, calidad y factibilidad de la fundamentación teórica y metodológica que se propone en la tesis.

\section{CONCLUSIONES}

La investigación realizada y expuesta en síntesis en la presente ponencia logró dar respuesta a los objetivos propuestos, en tal sentido puede concluirse lo siguiente:

1. La revisión y análisis de las fuentes bibliográficas sobre el tema confirma la necesidad y pertinencia de la formación en emprendimiento tomando como base para ello el cuerpo teórico de la cultura en emprendimiento como filosofía, enriquecido por los fundamentos conceptuales de la teoría educativa que ofrecen el enfoque histórico cultural y la teoría de las funciones sociales de la educación.
2. El análisis de la Constitución del Ecuador, del Plan Nacional de Desarrollo, de la Ley Orgánica de Educación Superior, del Reglamento del Régimen Académico, así como de discursos y presentaciones públicas de las figuras que dirigen hoy al país, han posibilitado constatar la necesidad de un tipo particular de graduado universitario, capaz de desarrollar proyectos de emprendimiento e innovación, que coadyuven a la transformación de la matriz productiva, tal y como se propone en el proyecto educativo objeto de estudio en la presente tesis.

3. Se demostró la pertinencia, solidez y posibilidades de aplicación del proyecto propuesto mediante el criterio de expertos, quienes de manera casi unánime reconocieron la fundamentación teórica del proyecto educativo de esta tesis como base sólida y cualitativamente superior para la formación en emprendimiento, con lo cual se alcanza una mirada holística, abarcadora y compleja, de la que carecía este tipo de formación, orientada hasta ahora mucho más a lo pragmático.

4. La fundamentación metodológica del proyecto fue igualmente avalada por los expertos, quienes convinieron en que las características y componentes del proyecto, en sus dos dimensiones, posibilitan su implementación y garantizan su flexibilidad y carácter dinámico en sus dos escenarios .

Las conclusiones hasta aquí expuestas evidencian la importancia y el valor de la investigación realizada y de la fundamentación del proyecto, cuya implementación debe dotar a los graduados de la Facultad de Especialidades Empresariales de una formación como emprendedores cualitativamente superior, a la altura de las necesidades que el Ecuador demanda en el momento actual.

\section{REFERENCIAS BIBLIOGRÁFICAS}

Aballe, V. Metodología de la Investigación educativa. Guayaquil: Edit UCSG.

Asamblea Nacional. (1998) Constitución Política de la República del Ecuador, R.O.\# 1,11 de agosto de 1998. Quito.

Asamblea Nacional (2009) Ley Orgánica de Educación Superior, R.O\# 77, de 15 de mayo del 2009. Quito. 
Arteaga, E. M. y Lasio V. (2009). Educación en emprendimiento en la universidad ecuatoriana: estado y oportunidades de mejora. Guayaquil: Escuela Superior Politécnica del Ecuador

Babson College y London Business School. (2006) Global Entrepreneurship Monitor. Londres.

Blanco Pérez, A. (2001) Introducción a la Sociología de la Educación. La Habana: Edit, Pueblo y Educación.

Druker, P. (1998), Su visión sobre La Administración, la Organización Basada en la Información, La Economía y La Sociedad. Bogotá: Editorial Norma.

Hidalgo, L. y Castro I. (2009). "La Pertinencia de Incorporar la Cultura del Emprendimiento en la Formación de los Abogados de la Carrera de Derecho de la Universidad Católica de Santiago de Guayaquil” Tesis de Maestría en Pensamiento Estratégico y Prospectiva para la Educación Superior, UCSG. Guayaquil

Kantis, H., Postigo, S., Mantis, J. F. y Tamborini, M. F. El surgimiento de emprendedores de base Universitaria: ¿en qué se diferencian? Evidencias Empíricas para el caso de Argentina. Accesible en: www.littec.ungs. edu.ar. Recuperado el 2 /09/2013.

Mantis, H. y Masahiko, I. (2002). Empresarialidad en Economías Emergentes: Creación y Desarrollo de Nuevas Empresas en América Latina y el Este de Asia, Banco Interamericano de Desarrollo.

Molina, D. (2013). Reporte de economía y Desarrollo 2013 de la CAF: "Emprendimientos en América Latina: desde la subsistencia hacia la transformación productiva”. Ministerio Coordinador de Producción, Empleo y Competitividad, Quito.

Ramon, S. (2009). Panorama del Emprendimiento en Ecuador y la situación de Loja Sandra ramon's Weblog. Sandra ramon's Weblog. Accesible en: de http://sandraramon. wordpress.com/2009/07/08/panorama-delemprendimiento-en-ecuador-y-la-situacionde-loja. Recuperado Agosto 31, 2012,

Rollin, K. (1993), La Evaluación de la Educación en América Latina: Una Comparación de Cinco Experiencias Nacionales. Buenos Aires: Edit. Centro de Estudios de Estado y Sociedad.

SENPLADES, (2013). Transformación de la matriz productiva. Gobierno de la Revolución Ciudadana. Quito.

Schumpeter, J. (1984). The Meaning of Rationality in the Social Sciences. Zeitschrift fur die gesamte Staatswissenschaft. Berlín.
Timmons, J. A., \& Spinelli, S. (2004). New Venture Creation Entrepreneurship for the 21st Century. New York: Edit. McGraw-Hill/Irwin.

Varela, R. (2004), La Educación, la Universidad y la Cultura Empresarial en América Latina: La Experiencia de la Universidad ICESI, http:// www.iadb.org/sds/doc/conf.6.24.04.varela.pdf

Vigotski, L.S. (1989) Obras Completas, tomo V. Editorial Pueblo y Educación. La Habana.

Unidades de análisis:

\section{POBLACIÓN Y MUESTRA}

Los métodos y técnicas declarados se aplicaron a una muestra representativa de estudiantes y graduados, así como a profesionales, autoridades, empresarios y profesores, los cuales constituyeron las unidades de análisis.

a. Egresados. Se encuestaron 102 egresados, graduados entre el 2008 y el 2013, el 55,9 $\%$ mujeres y el $44,1 \%$ hombres, sus edades fluctuaron en una media de 23 años.

b. Estudiantes. Las encuestas se realizaron a una muestra de 149 Estudiantes de la carrera de Emprendedores de la facultad de Especialidades Empresariales de la Universidad Católica de Santiago de Guayaquil (UCSC) $53,1 \%$ mujeres y $46,9 \%$ hombres, sus edades fluctuaron en una media de 19,7 años.

Para la selección del grupo a) Egresados, se tomó como población a los graduados de la carrera de Emprendedores del 2009 al 2012: 135. Muestra: 102

Para selección del grupo b) Estudiantes, se tomó como población a los estudiantes matriculados en el semestre B- 2011: 243. Muestra: 149

Para la selección de las muestras de los grupos a y b se aplicó la siguiente fórmula:

Fórmula para cálculo de muestra

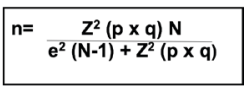

n= Tamaño de la Muestra

$\mathrm{N}=$ Tamaño de la Población

Z = Nivel de Confianza 95\% (valor de z proba-

bilidad normal de 1.96)

$\mathrm{e}=$ Porcentaje de error $(5 \%)$

$\mathrm{p}=$ Probabilidad de ocurrencia $(50 \%)$

$\mathrm{q}=$ Probabilidad de no ocurrencia $(50 \%)$

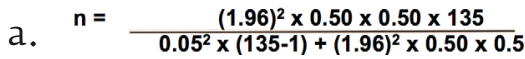

$\mathrm{n}=100,09$ personas a encuestar. 
b. $n=\frac{(1.96)^{2} \times 0.50 \times 0.50 \times 243}{0.05^{2} \times(243-1)+(1.96)^{2} \times 0.50 \times 0.50}$

$\mathrm{n}=149$ personas a encuestar.

c. Docentes: las encuestas se realizaron al total de 22 docentes de la carrera de Emprendedores de la Facultad de Especialidades Empresariales de la Univer- sidad Católica de Santiago de Guayaquil (UCSG), 72,7\% hombres y 27,3\% mujeres, sus edades fluctuaron en una media de 40 años, y con una media de 7,64 años de experiencia profesional.

d. Expertos: se seleccionó una muestra intencional de 15 expertos en el tema, procedentes de diversos sectores.

ANEXO 2

ESQUEMA DEL PROYECTO

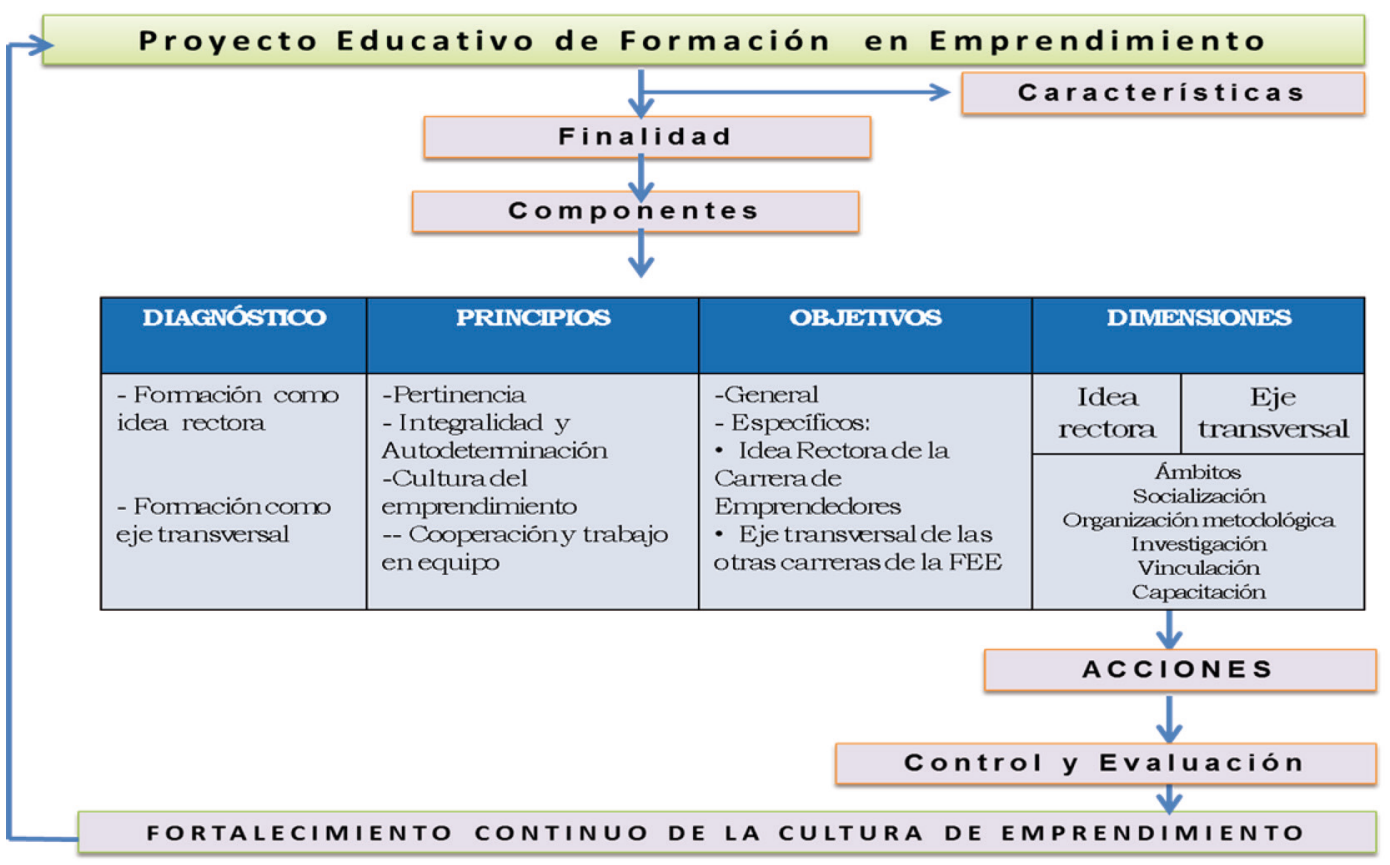

ANEXO 3

RESULTADOS DE LA VALIDACIÓN TEÓRICA

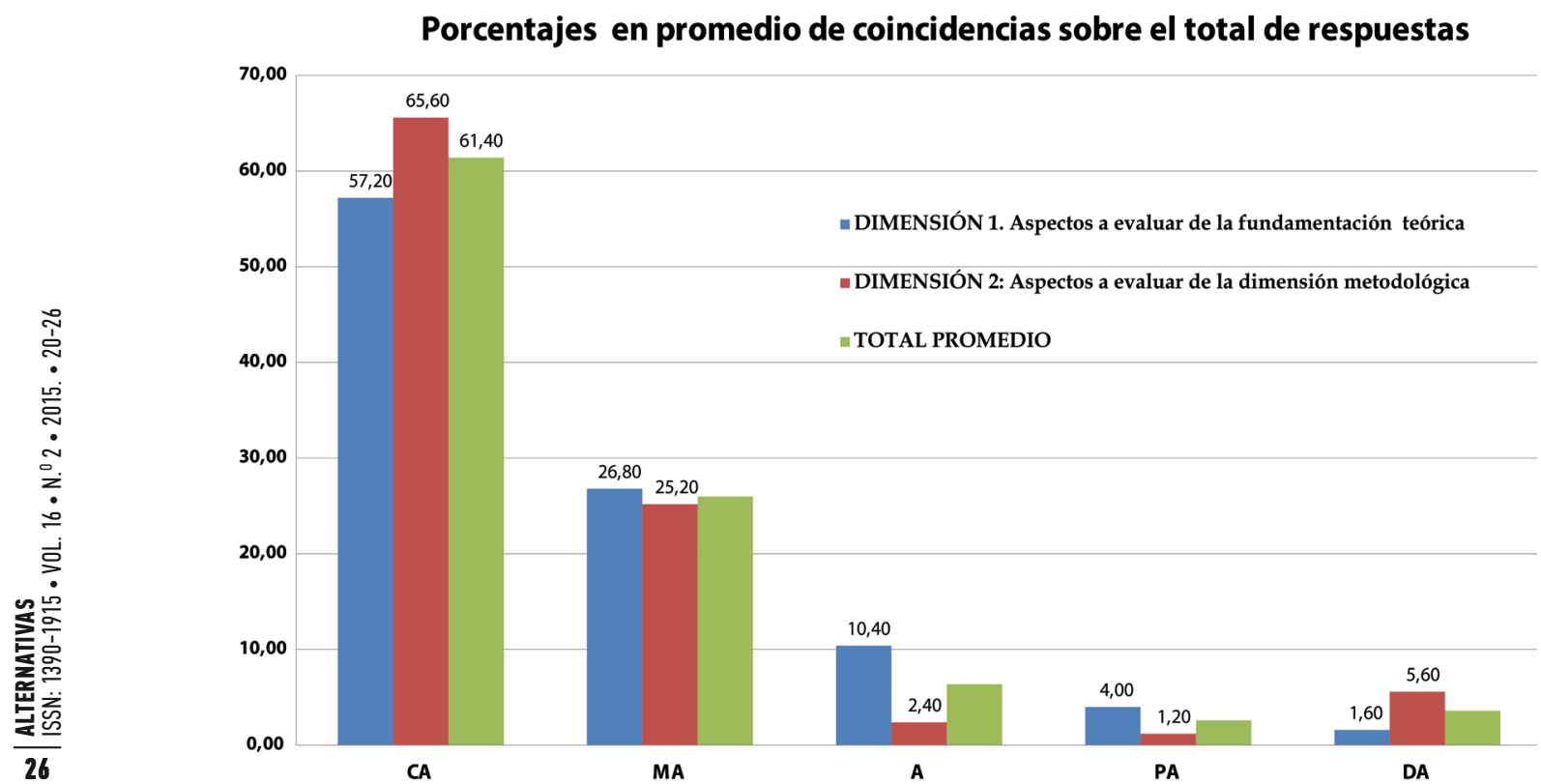

